\title{
The Use of Focused Ion Beam (FIB) Technology for Cell Wall Structure Elucidation
}

\author{
Melanie Blumentritt ${ }^{1}$, Scott D. Collins ${ }^{2}$ and Stephen M. Shaler ${ }^{3}$ \\ 1. Graduate Research Assistant, Advanced Structures and Composite Center, School of Forest \\ Resources, University of Maine, Orono, USA \\ 2. Professor of Chemistry, Laboratory for Surface Science and Technology, Department of \\ Chemistry, University of Maine, Orono, USA \\ 3. Associate Director, Advanced Structures and Composite Center, Professor of Wood Science, \\ School of Forest Resources, University of Maine, Orono, USA
}

The demand for sustainable products to meet current and emerging markets in construction, packaging, coatings, and chemicals is rapidly increasing due to economic, political, and environmental concerns. One approach to meet the markets for sustainable biomaterials is the development of a biorefinery. The primary focus of biorefinery research so far has been on extraction processes to both obtain a chemical feedstock for chemicals and fuels (primarily hemicelluloses, a group of C5 sugars found in wood and other plants) and modified wood for use in Oriented Strand Board (OSB) or pulp- and paper production. A key to this research is adjustment of process conditions and chemistries to remove valuable compounds without causing substantial degradation of the remaining material, ensuring a successful production of an established product (i.e. wood panels or paper products). While much is known about chemical processes and mechanisms of extraction, there has never been a direct microscopic evaluation of morphological changes within the wood cell wall structure, due to extraction. Morphological changes modify the integrity of the material and influence corresponding mechanical and physical properties that are important for the performance characteristics of the established product.

In our laboratory we explored the application potential of focused ion beam (FIB) technology, paired with scanning electron microscopy (SEM) for morphological observation, to precisely and accurately mill and section wood cells for a better understanding of the three dimensional architecture and topochemistry. We found that ion beam irrigation of a wood specimen resulted in the exposure of a skeletal like structure. The sputtering yield of a specimen, defined as the number of ejected particles per incident ion, is dependent on a number of parameters including beam current, incident angle, position of the collision cascade, surface binding energy, and the material. Material dependent properties are governed by the forces that bind the atoms of an element together as well as the atomic number and other periodic properties of the atoms. Wood, as other lignocellulosic materials, is chemically predominantly made of compounds built from carbon $(\mathrm{C})$, oxygen $(\mathrm{O})$, and hydrogen $(\mathrm{H})$ atoms. While cellulose and hemicellulose are linear polysaccharides, lignin has a more or less random three dimensional structure with a high amount of carbon double bonds $(\mathrm{C}=\mathrm{C})$. The bond energy of a double covalent bond is $\sim 130 \mathrm{kcal}$ and significantly higher than that of single bonds $(\mathrm{C}-\mathrm{C})$ at $\sim 85 \mathrm{kcal}$ [1]. Consequently, differences in sputtering yield of different parts of the wood cell wall are to be expected when comparing areas with a high amount of lignin (e.g. the middle lamella between cells) and areas dominated by cellulose. 
In order to confirm the assumption that the resulting skeletal structure of ion beam irrigation of wood is dependent on the chemical composition of the treated area it is necessary to identify the chemical compounds present. We choose to do this by selective electron dense staining of ultrathin wood sections with potassium permanganate $\left(\mathrm{KMnO}_{4}\right)$ which bonds to lignin [2-3]. Slivers of acetone extracted wood (Populus spec.) were embedded in Spurr's epoxy resin. After impregnation and curing, blocks were trimmed and faced off on an ultra-microtome (Leica EM UC6) using a glass knife followed by a diamond knife. Finally, blocks were mounted upright on SEM stubs and sputter coated before FIB milling. Squares were FIB milled on cell intersections at $30 \mathrm{kV}: 80 \mathrm{pA}$ for approximately 5 minutes per square (Fig.1). After FIB milling, $90 \mathrm{~nm}$ thick sections were cut from the blocks with a $35^{\circ}$ ultrasonic diamond knife (Diatome) and sections were mounted on grids. Since the milled areas could not support themselves the use of coated grids was necessary, which posed the subsequent challenge of getting sections to lie flat on the grids without excessive wrinkling of the sections that is an inherent problem of wood sections [4]. The sections were subsequently stained for $3 \mathrm{~min}$ with $\mathrm{KMnO}_{4}$ and examined with a transmission electron microscope (TEM Phillips CM10) at 100kV.

Figures $2 \mathrm{a}$ and $\mathrm{b}$ clearly show that the lignin rich middle lamella $(\mathrm{M})$ is less susceptible to FIB milling compared to the surrounding, more cellulose dominated, secondary cell wall (S). Future research will focus on better quantifying the milling results and to further develop specific techniques to use FIB/SEM on (modified) lignocellulosic materials for ultrastructural studies. We are planning to conduct serial sectioning with three dimensional reconstructions and a sputter rate study on wood model compound film to better understand the ion beam lignocellulosic specimen interactions [5].

[1] G Glockler, The Journal of Chemical Physics 19 (1951), p. 124-125.

[2] LADonaldson, IAWA Bulletin, 13 (1992), p. 381-387.

[3] J Fromm et al., Journal of Structural Biology, 143 (2003), p. 77-84.

[4] D Fengel, Wood Science and Technology, 1 (1967), p. 191-204.

[5] Kelly Edwards of the Electron Microscopy Laboratory at the University of Maine is thanked for his many useful discussions and contributions to this work.

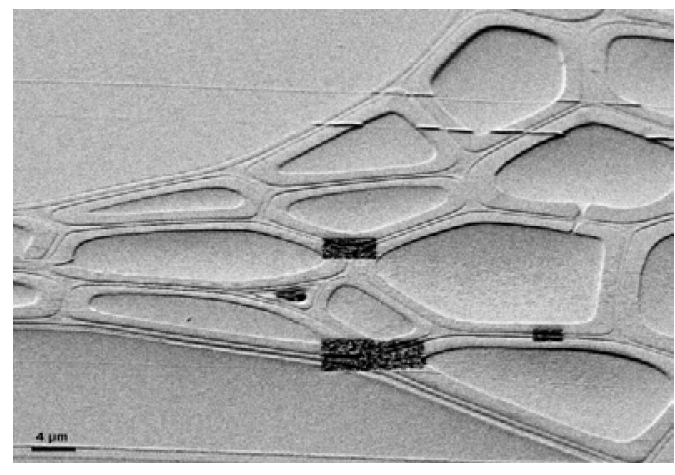

Figure 1. SEM $(5 \mathrm{kV})$ micrograph of a trimmed block face with four FIB milled squares (scale bar $4 \mu \mathrm{m}$ ).
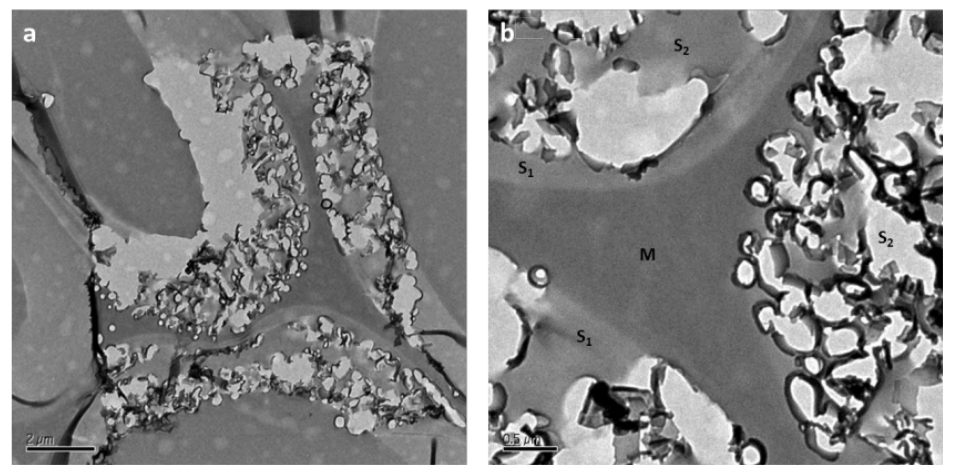

Figure 2. a) TEM micrograph of FIB milled square showing the retention of the middle lamella (scale bar $2 \mu \mathrm{m})$. b) Higher magnification micrograph of middle lamella (M) and surrounding more damaged secondary cell wall (S) layers (scale bar $0.5 \mu \mathrm{m}$ ). 